\title{
On Chaplygin Gas Braneworld Inflation with Monomial Potential
}

\author{
A. Safsafi $\mathbb{D}^{1},{ }^{1,2,3}$ I. Khay, ${ }^{1,2}$ F. Salamate,,$^{1,2}$ H. Chakir, ${ }^{1,2,3}$ and M. Bennai ${ }^{1,3}$ \\ ${ }^{1}$ Equipe Physique Quantique et Applications, Laboratoire de Physique de la Matière Condensée, \\ Faculté des Sciences Ben M'sik, Université Hassan II, Casablanca, Morocco \\ ${ }^{2}$ Equipe de Recherche Subatomique et Applications, Laboratoire de Physique de la Matière Condensée, \\ Faculté des Sciences Ben M'sik, Université Hassan II, Casablanca, Morocco \\ ${ }^{3}$ Groupement National de Physique des Hautes Energies, Focal Point, LabUFR-PHE, Rabat, Morocco
}

Correspondence should be addressed to A. Safsafi; safsafi_adil@yahoo.fr

Received 26 July 2017; Revised 1 October 2017; Accepted 4 October 2017; Published 25 June 2018

Academic Editor: Orlando Luongo

Copyright (C) 2018 A. Safsafi et al. This is an open access article distributed under the Creative Commons Attribution License, which permits unrestricted use, distribution, and reproduction in any medium, provided the original work is properly cited. The publication of this article was funded by SCOAP S $^{3}$

\begin{abstract}
We study the Chaplygin gas model as a candidate for inflation in the framework of the Randall-Sundrum type II braneworld model. We consider the original and generalized Chaplygin gas model in the presence of monomial potential. The inflationary spectrum perturbation parameters are reformulated and evaluated in the high-energy limit and we found that they depend on several parameters. We also showed that these perturbation parameters are widely compatible with the recent Planck data for a particular choice of the parameters space of the model. A suitable observational central value of $n_{s} \simeq 0.965$ is also obtained in the case of original and generalized Chaplygin gas.
\end{abstract}

\section{Introduction}

It is widely believed that the early universe underwent a period of accelerated expansion called inflation [1], which has become the standard paradigm of modern cosmology. Inflation model has been proposed as an attempt to solve the shortcoming of the standard Big-Bang model of cosmology, known usually as the flatness and the horizon problem [2]. Inflation is the candidate for understanding the physics of the very early universe, typing the evolution of the universe to the properties of one or more scalar inflaton fields, responsible for creating an accelerating expanding universe [3]. Recently, a great amount of work has been invested in studying the inflationary model with several candidates. Among these candidates is the Chaplygin gas model [4]. This model was also used to describe a mysterious dark sector, so-called dark energy and dark matter [5, 6], and the early universe [7]. In cosmology, dark energy is a form of unknown energy dominating the universe with a hugely negative pressure [8]. It is demonstrated by various astrophysical observations, including the accelerating universe [9]. Recall that the dark energy is defined by an exotic equation of state of the form $P_{\mathrm{DE}}=\omega \rho_{\mathrm{DE}}$, where $P_{\mathrm{DE}}$ and $\rho_{\mathrm{DE}}$ are the pressure and energy density of dark energy, while $\omega$ is the equation of state parameter of the dark energy [10]. There are several candidates to describe the dark matter and dark energy in the cosmology [11]. Among them are $K$-essence model [12], the $\Lambda$ CDM model [13], cosmological constant [14], tachyon model [15], and quintessence model [16]. There are different kinds of the Chaplygin gas model which have been proposed in the literature, for example, the general model named extended Chaplygin gas which is defined by an exotic equation of state of the form $[17,18]$

$$
p=\sum B_{m} \rho^{m}-\frac{A}{\rho^{\alpha}}
$$

where $B_{m}, A, m$ are universal positive constants and $0 \prec \alpha \preceq 1$. In recent years the extended Chaplygin gas was the subject of several cosmological and phenomenological studies $[19,20]$. Note that when $m=1$, we obtain the case of modified Chaplygin gas [21,22]. The original Chaplygin gas corresponds to the case of $B_{m}=0$ and $\alpha=1$; this type 
was studied in [23]. In the case of $B_{m}=0$ and $\alpha \neq 1$ it is known as the generalized Chaplygin gas (GCG) $[24,25]$ and finally for $B_{m}=0$ and $\alpha=0$ it corresponds to the $\Lambda$ CDM model [13]. Further, the Chaplygin gas models have been studied in different paper. In [26] the authors have studied the interaction of the dark energy with some fluids specially Chaplygin gas in the context of $f(T)$ theory. In [27], the authors have proposed an attempt for emergent universe scenario with modified Chaplygin gas and have shown that it is not possible to have emergent scenario with model. In another work [20], Pourhassan and Kahya have examined extended model of Chaplygin gas equation of state for which it recovers barotropic fluid with quadratic equation of state and have found that extended Chaplygin gas may be a more appropriate model than generalized and modified Chaplygin gas and give a best fit with the observational data.

On the other hand, the Chaplygin gas inspired inflation model [28] was the subject of several cosmological and phenomenological studies. In this context, the scalar field is usually the standard inflaton field, where the energy density can be extrapolated to obtain a successful inflationary period with a Chaplygin gas model. In the same context, a work has been done by Herrera [29] where the brane-Chaplygin inflationary model was studied in great detail and considered as a viable alternative model that can provide an accelerated expansion of the early universe. In extension of [29], similar work was performed for the case of the tachyon-Chaplygin inflationary model by using an exponential potential in the high-energy regime [30].

In the present paper, we are going to use the RandallSundrum II braneworld model to study the original and generalized Chaplygin gas as a candidate for the primordial inflation by assuming that the matter source on the brane consists of a Chaplygin gas. The Chaplygin gas emerges as an effective fluid of a generalized $d$-brane in a $(d+1,1)$ spacetime, where the action can be written as a generalized Born-Infeld action [25]. These models have been extensively studied in the literature [7]. The motivation for introducing Chaplygin-brane scenarios is the increasing interest in higher-dimensional cosmological models, motivated by superstring theory, where the matter fields are confined to a lower-dimensional brane while gravity can propagate in the bulk. On the other hand, the Chaplygin gas model seems to be a viable alternative to models that provide an accelerated expansion of the early universe. Our aim is to quantify the modifications of the Chaplygin inspired inflation in the braneworld scenario. We use the monomial potential to study various perturbation spectrum parameters such as the scalar spectral index $n_{s}$ and the ratio $r$ and the running of the scalar spectral index $d n_{s} / d \ln (k)$ in the high-energy limit, particularly for a suitable choice of the different parameters. We show that the inflation parameters are in good agreement with recent Planck 2015 data [31].

An outline of the remainder of this paper is as follows: We first begin in Section 2, by recalling the standard inflation and Chaplygin gas braneworld inflation formalism, in particular the modified Friedmann equation. In Section 3, we study different perturbation spectrum concerning monomial potential in the high-energy limit, and we present our results for original and generalized Chaplygin gas on the brane. The last section is devoted to conclusion.

\section{Generalized Chaplygin Gas Braneworld Inflation}

2.1. Inflationary Universe. In this section we propose a short description on standard inflation formalism to enable readers to understand the meanings of terms here involved. Inflation is the period of the early universe that undergoes an accelerating phase [1]. This period is equivalent to $\ddot{a}>$ 0 . The candidate that can yield this acceleration phase and is responsible for driving inflation is a scalar field named inflaton field. The pressure and energy of the inflaton field are given by $p_{\phi}=(1 / 2) \dot{\phi}^{2}-V(\phi)$ and $\rho_{\phi}=(1 / 2) \dot{\phi}^{2}+V(\phi)$, where $V(\phi)$ is the scalar potential. The Friedmann equation reads $H^{2}=(8 \pi G / 3)\left[(1 / 2) \dot{\phi}^{2}+V(\phi)\right]$. During inflation, the potential $V(\phi)=V$ depends only on the inflaton field $\phi$. It is supposed that the field equation $\ddot{\phi}+3 H \dot{\phi}=-V^{\prime}(\phi)$ is well-approximated by $3 H \dot{\phi}=-V^{\prime}(\phi)$. The condition for acceleration requires that $\dot{\phi}^{2} \prec V$. This is called the slow-roll approximation. With very small $\dot{\phi}^{2}$, the Friedmann equation is approximately $3 M_{p}^{2} H^{2} \simeq V$, and the flatness conditions $\varepsilon \ll 1$ and $|\eta| \ll 1$, where $\varepsilon=(1 / 2) M_{p}^{2}\left(V^{\prime} / V\right)^{2}$ and $\eta=$ $M_{p}^{2}\left(V^{\prime \prime} / V^{\prime \prime}\right)$. The amount of inflation can be measured in terms of the $e$-folding number $N$ given by equation $N=$ $M_{p}^{-2} \int_{\phi_{\text {end }}}^{\phi_{*}}\left(V / V^{\prime}\right) d \phi$, where $\phi_{*}$ and $\phi_{\text {end }}$ are the values of the scalar field at the epoch when the cosmological scales exit the horizon and at the end of inflation, respectively.

The small quantum fluctuations in the scalar field lead to fluctuations in the energy density which was studied in a perturbative theory [32]. As discussed in [33] quantum fluctuations effects of the inflaton are generally negligible, since the coupling of the scalar field to bulk gravitational fluctuations only modifies the usual $4 \mathrm{D}$ predictions at the next order in the slow-roll expansion. So, one can define the power spectrum of the curvature perturbations as $P_{R}(k)=$ $\left(H^{2} / 2 \pi \phi\right)^{2}$. On the other hand, the quantum fluctuations in the scalar field lead also to fluctuations in the metric [34]. In this way, one can define the amplitude of tensor perturbations as $P_{g}(k)=\left(8 / M_{p}^{2}\right)(H / 2 \pi)^{2} F^{2}(x)$. These results lead to the ratio of tensor-to-scalar perturbations $r=P_{g}(k) / P_{R}(k)$. In relation to $P_{R}(k)$, the scalar spectral index is defined as $n_{s}-1=$ $d \ln P_{R}(k) / d \ln (k)$. Reference [3] is recommended for further reading on inflation.

2.2. Generalized Chaplygin Gas on the Brane. Braneworld inflation [35] is a particular kind of inflation models. It is based primarily on the cosmological model RandallSundrum type II which describes the universe in five dimensions with the presence of a brane that includes all ordinary matter. The generalized Chaplygin gas is a perfect fluid characterized by the following equation of state [36]:

$$
p=-\frac{A}{\rho^{\alpha}},
$$


where $\rho$ and $p$ are the energy density and pressure of the generalized Chaplygin gas, respectively, $\alpha$ is a constant satisfying $0 \prec \alpha \preceq 1$, and $A$ is a positive constant.

Inserting (2) in the equation of conservation of energy $\dot{\rho}+$ $3 H(\rho+p)=0$, we obtain the following expression for the energy density:

$$
\rho_{\mathrm{ch}}=\left[A+\left(\rho_{\mathrm{ch} 0}^{\alpha+1}-A\right)\left(\frac{a_{0}}{a}\right)^{3(\alpha+1)}\right]^{1 /(\alpha+1)},
$$

where $a_{0}$ and $\rho_{\text {ch } 0}$ are the current values of the scale factor and the generalized Chaplygin gas energy density, respectively.

The modification of (5) is realized from an extrapolation of (3), where the density matter $\rho_{m} \sim a^{-3}$ is replaced by the scalar field as $\rho=\left[\mathrm{A}+\rho_{m}^{(\alpha+1)}\right]^{1 /(\alpha+1)} \rightarrow \rho=\left[A+\rho_{\phi}^{(\alpha+1)}\right]^{1 /(\alpha+1)}$.

In this section, we will recall briefly some basic facts of Randall-Sundrum type II braneworld model $[37,38]$. We suppose that the universe is filled with a perfect fluid with energy density $\rho(t)$ and pressure $p(t)$ in which the Friedmann equation is modified from its usual form [29]

$$
H^{2}=k \rho_{\phi}\left[1+\frac{\rho_{\phi}}{2 \lambda}\right]+\frac{\Lambda_{4}}{3}+\frac{\xi}{a^{4}},
$$

where $H=\dot{a} / a$ defines the Hubble parameter, $\rho_{\phi}$ represents the matter confined to the brane, $k=8 \pi G / 3=8 \pi / 3 M_{p}^{2}, \Lambda_{4}$ is the current cosmological constant, and $\xi$ is an integration constant and thus transmitting bulk graviton influence onto the brane. This term appears as a form of "dark radiation" and may be fixed by observation [39]. However, during inflation this term is rapidly diluted, so we will neglect it, where $\lambda$ is the brane tension and $M_{p}$ is the four-dimensional Planck mass, which is related to the five-dimensional $M_{5}$ by $M_{p}=$ $\sqrt{3 M_{5}^{6} / 4 \pi \lambda}$. Note that the crucial correction to standard inflation is given by the density quadratic term $\rho^{(\alpha+1)}$. Note also that, in the limit $\lambda \rightarrow \infty$, we recover standard fourdimensional general relativistic results.

The Friedmann equation will become [30]

$$
\begin{aligned}
H^{2}= & \frac{8 \pi}{3 M_{p}^{2}}\left(A+\rho_{\phi}^{(\alpha+1)}\right)^{1 /(\alpha+1)} \\
& \cdot\left[1+\frac{\left(A+\rho_{\phi}^{(\alpha+1)}\right)^{1 /(\alpha+1)}}{2 \lambda}\right] .
\end{aligned}
$$

In four-dimensional general relativity, the condition for inflation is $\dot{\phi}^{2} \ll V(\phi)$, that is, $p_{\phi} \prec-(1 / 3) \rho_{\phi}$, where $p_{\phi}=$ $(1 / 2) \dot{\phi}^{2}-V(\phi)$ and $\rho_{\phi}=(1 / 2) \dot{\phi}^{2}+V(\phi), V(\phi)=V$ is the scalar potential, and $\phi$ is the inflaton field; the scalar field satisfies the Klein-Gordon equation:

$$
\ddot{\phi}+3 H \dot{\phi}+V^{\prime}(\phi)=0
$$

Note that $\dot{\phi}=\partial \phi / \partial t, \ddot{\phi}=\partial^{2} \phi / \partial t^{2}$, and $V^{\prime}=\partial V / \partial \phi$. During inflation, the relation between the energy density and the scalar potential is $\rho_{\phi} \simeq V$; we consider the slow-roll approximations $\dot{\phi}^{2} \ll V(\phi)$ and $\ddot{\phi} \ll 3 H \dot{\phi}$; the Friedmann equation reduces to

$$
\begin{aligned}
H^{2}= & \frac{8 \pi}{3 M_{p}^{2}}\left(A+V^{(\alpha+1)}\right)^{1 /(\alpha+1)} \\
& \cdot\left[1+\frac{\left(A+V^{(\alpha+1)}\right)^{1 /(\alpha+1)}}{2 \lambda}\right] .
\end{aligned}
$$

The term in square brackets is the brane-modification to the standard slow-roll expression for the Hubble rate.

We consider the slow-roll parameters, in the RandallSundrum type II braneworld model [33]. The two first parameters are given for generalized Chaplygin gas model by

$$
\begin{aligned}
\varepsilon= & \frac{M_{P}^{2}}{16 \pi} \\
& \cdot \frac{V^{\alpha} V^{\prime 2}}{\left(A+V^{(\alpha+1)}\right)^{(\alpha+2) /(\alpha+1)}}\left[\frac{1+\left(A+V^{(\alpha+1)}\right)^{1 /(\alpha+1)} / \lambda}{\left(1+\left(A+V^{(\alpha+1)}\right)^{1 /(\alpha+1)} / 2 \lambda\right)^{2}}\right], \\
\eta= & \frac{M_{P}^{2}}{8 \pi} \frac{V^{\prime \prime}}{\left(A+V^{(\alpha+1)}\right)^{1 /(\alpha+1)}}\left[\frac{1}{1+\left(A+V^{(\alpha+1)}\right)^{1 /(\alpha+1)} / 2 \lambda}\right] .
\end{aligned}
$$

The inflationary phase ends when $\varepsilon$ or $|\eta|$ is equal to one; during inflation, the conditions $\varepsilon \ll 1$ and $|\eta| \ll 1$ are satisfied. Note that, at low energies, $V \ll \lambda$, the slow-roll parameters reduce to the standard inflation.

In addition, the number of $e$-foldings is given by

$$
\begin{aligned}
N= & \frac{-8 \pi}{M_{P}^{2}} \int_{V_{*}}^{V_{\text {end }}} \frac{\left(A+V^{(\alpha+1)}\right)^{1 /(\alpha+1)}}{V^{\prime 2}} \\
& \cdot\left[1+\frac{\left(A+V^{(\alpha+1)}\right)^{1 /(\alpha+1)}}{2 \lambda}\right] d V,
\end{aligned}
$$

where $V_{*}$ and $V_{\text {end }}$ are the values of the potentials at the horizon exit and the end of inflation, respectively.

The inflationary spectrum perturbation is produced by quantum fluctuations of fields around their homogeneous background values. The small quantum fluctuations in the scalar field lead to fluctuations in the energy density and in the metric; for that, we define the power spectrum of the curvature perturbations by [33]

$$
P_{r}(k)=\left(\frac{H^{2}}{2 \pi \dot{\phi}}\right)^{2} .
$$

By using (7) and (10), we find the expression for power spectrum of the curvature perturbations:

$$
\begin{aligned}
& P_{r}(k)=\frac{128 \pi}{3 M_{p}^{6}} \\
& \cdot \frac{\left(A+V^{(\alpha+1)}\right)^{3 /(\alpha+1)}}{V^{\prime 2}}\left[1+\frac{\left(A+V^{(\alpha+1)}\right)^{1 /(\alpha+1)}}{2 \lambda}\right]^{3} .
\end{aligned}
$$


Another important inflationary spectrum parameter is the amplitude of the tensorial perturbations $P_{g}(k)$, describing the primordial gravitational wave perturbations produced by a period of extreme slow-roll inflation, which is defined by [34]

$$
P_{g}=\frac{64 \pi}{M_{p}^{2}}\left(\frac{H}{2 \pi}\right)^{2} F^{2}(x),
$$

where $x=H M_{p} \sqrt{3 / 4 \pi \lambda}$ and $F^{2}(x)=\left(\sqrt{1+x^{2}}-\right.$ $\left.x^{2} \sinh ^{-1}(1 / x)\right)^{-1}$. Note that, in the low-energy limit $(A+$ $\left.V^{(\alpha+1)}\right)^{1 /(\alpha+1)} \ll \lambda$, we have $F^{2}(x) \simeq 1$ and, in the high-energy $\operatorname{limit}\left(A+V^{(\alpha+1)}\right)^{1 /(\alpha+1)} \gg \lambda, F^{2}(x) \simeq(3 / 2) x=(3 / 2)((A+$ $\left.\left.V^{(\alpha+1)}\right)^{1 /(\alpha+1)} / \lambda\right)$.

We define the tensor-to-scalar ratio $r$ as

$$
r=\left(\frac{P_{g}(k)}{P_{r}(k)}\right)_{k=k^{*}} .
$$

Here $k^{*}$ corresponds to the case $k=H a$, the value when the universe scale crosses the Hubble horizon during inflation. From (11) and (12), the tensor-to-scalar ratio is given by

$$
\begin{aligned}
r= & \frac{M_{p}^{2}}{\pi} \\
& \cdot \frac{V^{\prime 2} F^{2}(x)}{\left(A+V^{(\alpha+1)}\right)^{2 /(\alpha+1)}\left(1+\left(A+V^{(\alpha+1)}\right)^{1 /(\alpha+1)} / 2 \lambda\right)^{2}} .
\end{aligned}
$$

The scalar spectral index is presented by [32]

$$
\begin{aligned}
n_{s} & -1=\frac{d \ln P_{R}(k)}{d \ln (k)} \\
& =\frac{M_{p}^{2}}{8 \pi\left(A+V^{(\alpha+1)}\right)^{1 /(\alpha+1)}\left(1+\left(A+V^{(\alpha+1)}\right)^{1 /(\alpha+1)} / 2 \lambda\right)}(-3 \\
& \left.\cdot \frac{V^{\alpha} V^{\prime 2}}{\left(A+V^{\alpha+1}\right)} \frac{\left(1+\left(A+V^{(\alpha+1)}\right)^{1 /(\alpha+1)} / \lambda\right)}{\left(1+\left(A+V^{(\alpha+1)}\right)^{1 /(\alpha+1)} / 2 \lambda\right)}+2 V^{\prime \prime}\right) .
\end{aligned}
$$

The running of the scalar index is also defined as

$$
\begin{aligned}
& \frac{d n_{s}}{d \ln (k)}=\frac{M_{p}^{2}}{4 \pi} \frac{V^{\prime}}{\left(A+V^{(\alpha+1)}\right)^{1 /(\alpha+1)}} \\
& \cdot \frac{1}{\left(1+\left(A+V^{(\alpha+1)}\right)^{1 /(\alpha+1)} / 2 \lambda\right)}\left(3 \frac{\partial \varepsilon}{\partial \phi}-\frac{\partial \eta}{\partial \phi}\right) .
\end{aligned}
$$

Note that, in the limit $A \rightarrow 0$, the perturbation spectrum parameters coincide with brane inflation [33], in particular for $\alpha=1$. Also, in the low-energy limit, $\left(A+V^{(\alpha+1)}\right)^{1 /(\alpha+1)} \ll$ $\lambda$, the slow-parameters reduce to the standard form [28].

In what follows, we shall apply the above braneworld formalism with a monomial potential in the high-energy limit; that is, $\left(A+V^{(\alpha+1)}\right)^{1 /(\alpha+1)} \gg \lambda$, in relation to recent Planck data.

\section{Chaplygin Gas with Monomial Potential}

3.1. Original Chaplygin Gas. In the following, we will concentrate on the original Chaplygin gas; it is reached as a special case of the general Chaplygin gas and is proposed as possible explanation of the acceleration of the current universe. The original Chaplygin gas model has been extensively studied. For example, [40] studied the behaviour of density perturbations in a universe dominated by the Chaplygin gas and found that, in spite of presenting negative pressure of Chaplygin gas, it is stable at small scale, opposite to in general what happens with perfect fluids with negative pressure. In [23], the authors have focused on studying a Chaplygin gas model in braneworld inflation with an exponential potential and have obtained that, for negligible and small running of the scalar spectral index, the inflationary parameters are in good agreement with observation data. In another example [41], the authors have analysed a phase space of the evolution for a Friedmann Robertson Walker universe driven by an interaction of Chaplygin gas and dark matter; their results are derived from continuity equations, which means that they are independent of any theories of gravity. The original Chaplygin gas model is characterized by an exotic equation of state of the form

$$
p=-\frac{A}{\rho},
$$

where $A$ is a positive constant.

In this section we will propose to investigate monomial potential in braneworld context with generalized Chaplygin gas. This potential was used in a very recent model in different work. In the paper [42] the authors study the attractors solutions of the dynamical system of a scalar field endowed with monomial potentials and show that the behaviour found for monomial potentials is typical in realistic inflationary models. Furthermore, Zarrouki et al. have studied various inflationary spectrum perturbation parameters with three types of potentials; they have shown that the monomial potential provides the best fit results to observations data [43]. This potential is given by

$$
V=M \phi^{n}
$$

where $n$ is a positive integer and $M$ is a parameter of dimension $[E]^{4-n}$. In order to derive the inflationary parameters $n_{s}$, $r$, and $d n_{s} / d \ln (k)$, let us consider the monomial potential. In this case, the scalar spectral index $n_{s}$, the ratio $r$, and the running of the scalar index $d n_{s} / d \ln (k)$ become

$$
\begin{aligned}
n_{s} & =\frac{M_{p}^{2} \lambda}{2 \pi\left(A+M^{2} \phi_{*}^{2 n}\right)}\left(-\frac{3 n^{2} M^{3} \phi_{*}^{3 n-2}}{\left(A+M^{2} \phi_{*}^{2 n}\right)}\right. \\
& \left.+n(n-1) M \phi_{*}^{n-2}\right)+1,
\end{aligned}
$$




$$
\begin{aligned}
& r=\frac{6 M_{p}^{2} \lambda M^{2} n^{2} \phi_{*}^{2 n-2}}{\pi\left(A+M^{2} \phi_{*}^{2 n}\right)^{3 / 2}}, \\
& \frac{d n_{s}}{d \ln (k)}=-\frac{M_{p}^{4} \lambda^{2} n^{2}(2 n+1)(n+2)}{8 \pi^{2} \phi_{*}^{4}\left(A+\left(M \phi^{n}\right)^{2}\right)} .
\end{aligned}
$$

Note that, in the limit $A \rightarrow 0$, the scalar spectral index $n_{s}$, the ratio $r$, and $d n_{s} / d \ln (k)$ coincide with those in [43].

Although we have analytic results for slow-roll parameters and the number of $e$-foldings, it is not easy to solve them to obtain $\phi_{*}$ at which the observables $n_{s}, r$, and $d n_{s} / d \ln (k)$ should be evaluated. For this, we proceed numerically by finding $\phi_{\text {end }}$ and using (9) to obtain $\phi_{*}$ while making sure that the slow-roll parameters remain small in this range of $\phi$.

To complete our study with original Chaplygin gas, we analyse the variations of the perturbation spectrum parameters with respect to $N$ for various values of $n=$ $1,2,3,4$. We can see that these observables depend on several parameters. For this purpose, we take the inflationary scale $M \sim O\left(10^{15} \mathrm{GeV}\right)$, the brane tension value $\lambda \sim O$ $\left(10^{68} \mathrm{GeV}^{4}\right)$, and $A \sim 10^{-13} M_{p}^{8}$ [29], in order to obtain consistent perturbation spectrum parameters with recent Planck data.

Figure 1 presents the variations of scalar spectral index $n_{s}$ as a function of $e$-folding number $N$. The scalar spectral index $n_{s}$ has an increasing behaviour as we increase the $e$-folding number $N$. We also note that the values of $n_{s}$ are found to be consistent with the Planck data for large domain of $N$ and the central value of $n_{s} \simeq 0.965$ is obtained in particular for $n=1 ; 2$.

In Figure 2 we have plotted $r$ as a function of $e$-folding number $N$ for different values of $n$. Generally, the ratio $r$ has a decreasing behaviour with respect to $N$. We remark also that, in order to confront $r$ with Planck data, we must have large values of $N$ for the four values of $n$.

Figure 3 shows that the observable $d n_{s} / d \ln (k)$ is an increasing function with respect to $N$. We have obtained extremely weak values which are consistent with Planck data and concluded that they get smaller as we increase the values of $n$.

To summarize this subsection, we have found that the results reviewed in the context of the original Chaplygin gas model are compatible with the latest observational measurements for a particular choice of $e$-folding number $N$ and constant values of $n$.

In the following, we will study the case of generalized Chaplygin gas with $\alpha \neq 1$. We will discuss the effect of introducing the constant $\alpha$ on the perturbation spectrum of the model. Our results will be compared to observations and we will show that the inflation can occur successfully in relation to recent observations.

3.2. Generalized Chaplygin Gas. It is well known that generalized Chaplygin gas is one of the most natural candidates of dark energy models to explain the accelerated expansion of the universe. In this context, these models have been

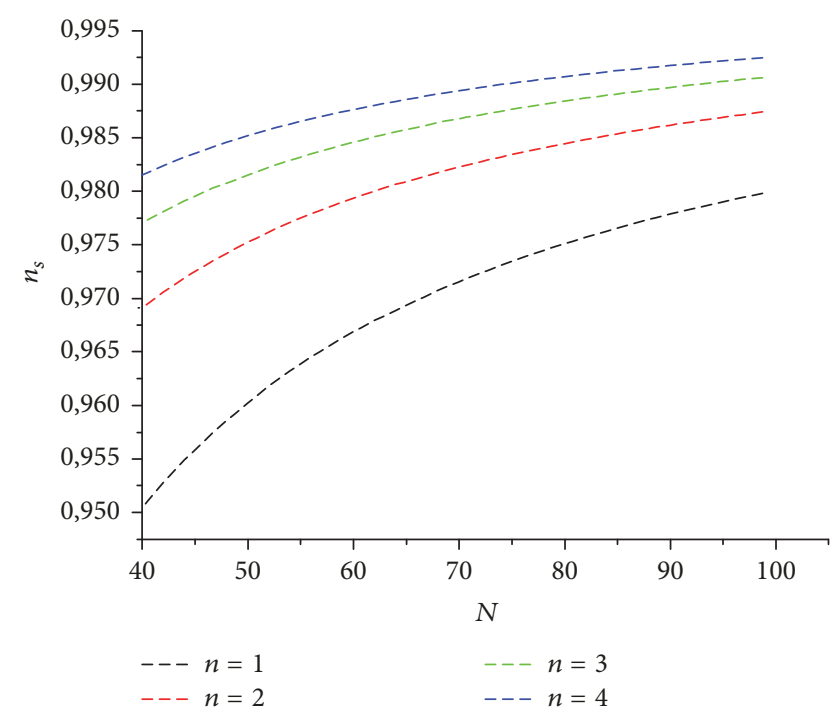

FIgURE 1: $n_{s}$ versus $N$ for various values of $n$. We take $M \sim$ $O\left(10^{15} \mathrm{GeV}\right), \lambda \sim O\left(10^{68} \mathrm{GeV}^{4}\right)$, and $A \sim 10^{-13} \mathrm{M}_{p}^{8}$.

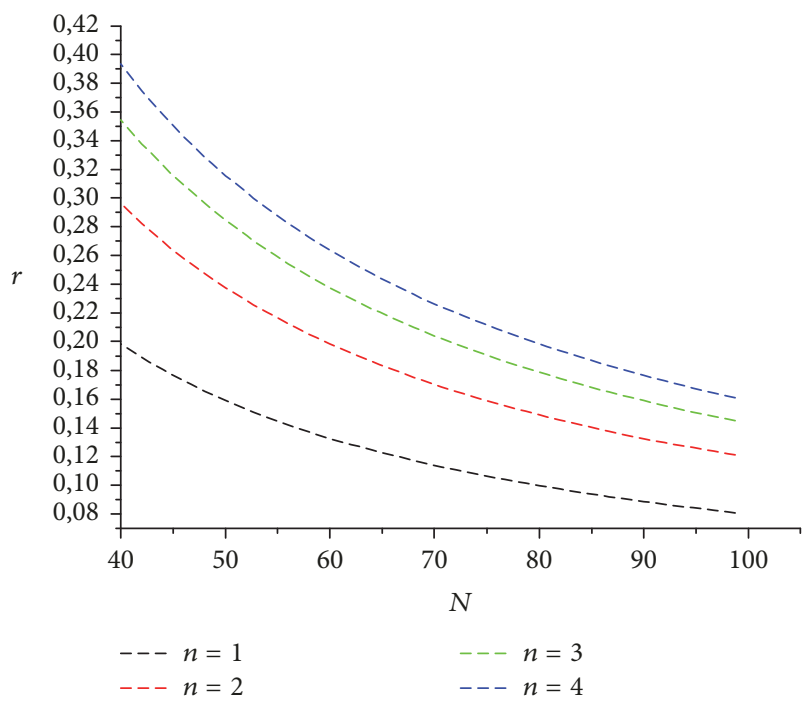

FIgURE 2: $r$ versus $N$ for various values of $n$. We take $M \sim$ $O\left(10^{15} \mathrm{GeV}\right), \lambda \sim O\left(10^{68} \mathrm{GeV}^{4}\right)$, and $A \sim 10^{-13} M_{p}^{8}$.

extensively studied in the literature. Moreover, in the work [44] the authors have studied an inflationary scenario in the presence of generalized Chaplygin gas in the light of the Planck and BICEP2 experiments and have obtained the constraints on $n_{s}$ and $r$. In another work [45] the authors have examined the effect of anisotropy on generalized Chaplygin gas scalar field and its interaction with other dark energy models; they concluded that the increase in anisotropy leads to more correspondence between the dark energy scalar field model and observational data. On the other hand Herrera et al. have considered an intermediate inflationary universe model in the context of a generalized Chaplygin gas in the slow-roll approximation and have shown that the intermediate generalized Chaplygin gas inflationary 


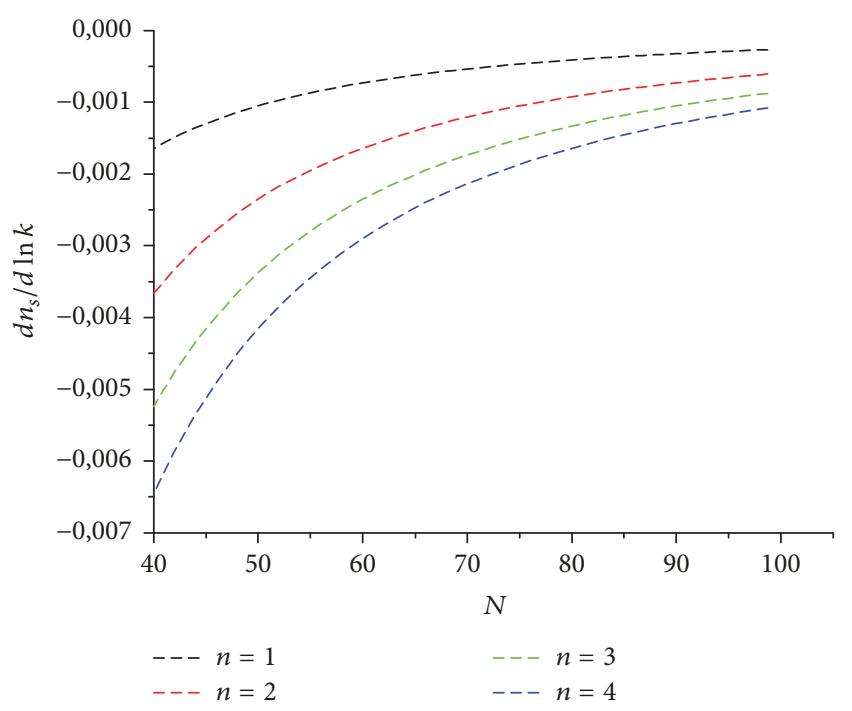

FIgURE 3: $d n_{s} / d \ln (k)$ versus $N$ for various values of $n$. We take $M \sim$ $O\left(10^{15} \mathrm{GeV}\right), \lambda \sim O\left(10^{68} \mathrm{GeV}^{4}\right)$, and $A \sim 10^{-13} M_{p}^{8}$.

models are less restricted than analogous ones, standard intermediate inflationary models, due to the introduction of $\alpha$ and $\beta$ parameters [46]. In the context of brane-inflationary background, the generalized cosmic Chaplygin model was studied by Jawad et al. [47] in the presence of chaotic potential in the high-energy limit; various inflationary parameters were evaluated and compared by Planck data.

In this part, we study the generalized Chaplygin gas model in the presence of monomial potential, using the basic formalism obtained in Section 2 in the context of high-energy limit. In this case, the scalar spectral index $n_{s}$, the ratio $r$, and the running of the scalar index $d n_{s} / d \ln (k)$ become

$$
\begin{aligned}
n_{s} & =\frac{M_{p}^{2} \lambda}{2 \pi\left(A+\left(M \phi_{*}^{n}\right)^{\alpha+1}\right)^{2 /(\alpha+1)}}\left(-\frac{3 n^{2} M^{3} \phi_{*}^{3 n-2}}{\left(A+\left(M \phi_{*}^{n}\right)^{\alpha+1}\right)^{1 /(\alpha+1)}}\right. \\
& \left.+n(n-1) M \phi_{*}^{n-2}\right)+1 .
\end{aligned}
$$

The tensor-to-scalar ratio $r$ will be given by

$$
r=\frac{6 M_{p}^{4} \lambda M^{2} n^{2} \phi_{*}^{2 n-2}}{\pi\left(A+\left(M \phi_{*}^{n}\right)^{\alpha+1}\right)^{3 /(\alpha+1)}} .
$$

The running of the scalar spectral index will be in the following form:

$$
\frac{d n_{s}}{d \ln (k)}=-\frac{M_{p}^{4} \lambda^{2} n^{2}(2 n+1)(n+2)}{8 \pi^{2} \phi_{*}^{4}\left(A+\left(M \phi_{*}^{n}\right)^{\alpha+1}\right)^{2 /(\alpha+1)}} .
$$

We note that, as in the previous case, the inflaton value before the end of inflation $\phi_{*}$ can be obtained numerically from (9).

Based on the above formulas and to confront simultaneously the observables $n_{s}, r$, and $d n_{s} / d \ln (k)$ with observations,

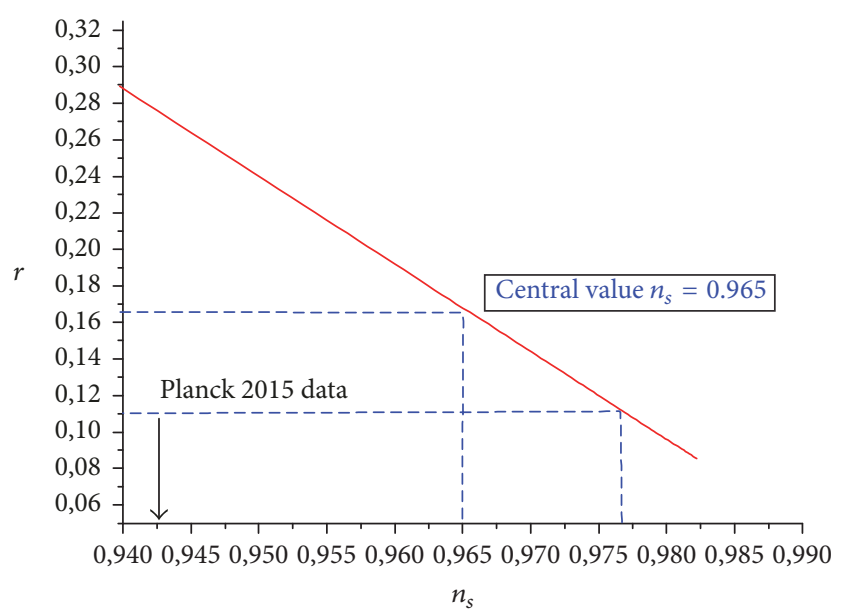

FIGURE 4: Evolution of $r$ versus $n_{s}$ for various values of $N$. We take $M \sim O\left(10^{15} \mathrm{GeV}\right), \lambda \sim O\left(10^{68} \mathrm{GeV}^{4}\right), A \sim 10^{-13} M_{p}^{8}$, and $n=2$.

we study the relative variation of these parameters. We can see that these observables depend on several parameters. Therefore, as the previous case we take the inflationary scale $M \sim O\left(10^{15} \mathrm{GeV}\right)$, the brane tension value $\lambda \sim$ $O\left(10^{68} \mathrm{GeV}^{4}\right)$ and $A \sim 10^{-13} M_{p}^{8}[29]$, and $n=2$ which corresponds to chaotic case.

We will discuss some values of the above inflationary parameters in relation to the $e$-folds number $N$ and the parameter $\alpha$. The central region, given by the Planck data, of the spectral index $n_{s} \in[0.959 ; 0.969]$ gives $45<N<75$ for the values of $\alpha$ in order of $\alpha \sim O\left(10^{-3}\right)-O\left(10^{-1}\right)$. Regarding the ratio of scalar-to-tensor curvature perturbation, the Planck constraint $r<0.11$, which requires large values of the folding number; that is, $N>80$, and for $d n_{s} / d \ln (k) \epsilon$ [ $-0.0166 ;-0.0039]$, we get $56<N<150$ for large domain of value of $\alpha$ within $0<\alpha<1$. This shows that parameter $\alpha$ has a small influence on the inflationary observables compared to the original Chaplygin gas. But we can have the normal value which is most commonly used for the $e$-folding number, required for solving the horizon and flatness problems.

Figure 4 shows the variation of $n_{s}$ with respect to ratio $r$; we remark that $n_{s}$ is a decreasing linear function with $r$. The ratio $r$ is compatible with Planck data where $r<0.11$ corresponds to $N>80$ and $n_{s} \geq 0.975$. The central value $n_{s} \simeq 0.965$, where $N \sim 65$ corresponds to the ratio $r \sim 0.17$.

Figure 5 presents the running of the scalar spectral index $d n_{s} / d \ln (k)$ as function of $n_{s}$. We see that $d n_{s} / d \ln (k)$ increases with respect to $n_{s}$. The central value of the scalar spectral index $n_{s} \simeq 0.965$ corresponds to $N=65$, giving $d n_{s} / d \ln (k) \simeq-0.0005$, which is consistent with Planck data.

In Figure 6 we have plotted the ratio $r$ according to the running of the scalar spectral index $d n_{s} / d \ln (k)$, which is a decreasing function with the variation of $N$. The tensor-toscalar ratio given by Planck corresponds to $N>80$ which gives a domain of the running $\left(d n_{s} / d \ln (k)\right) \preceq-0.00024$.

To summarize this subsection, our numerical calculation shows that, for a particular choice of the parameters of the model, the obtained results are compatible with the Planck 


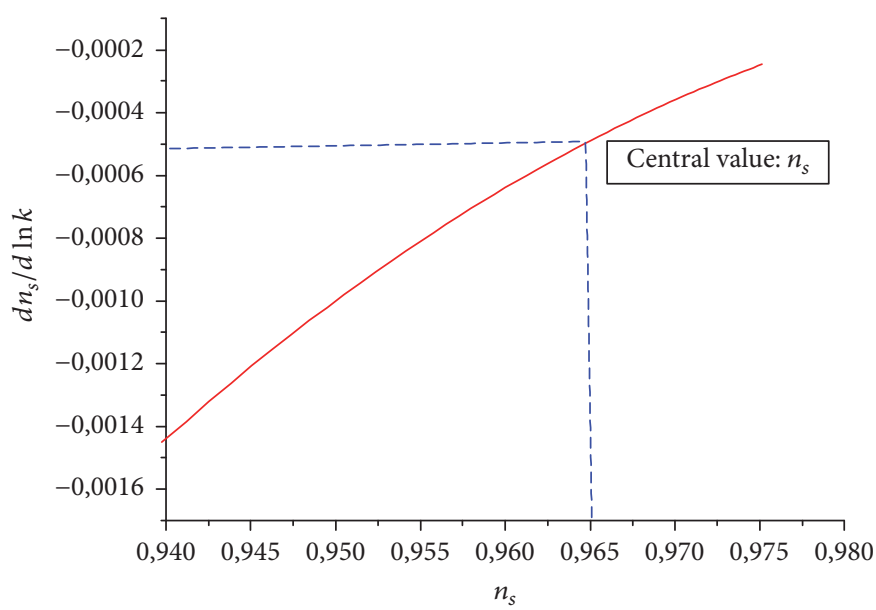

FIGURE 5: Evolution of $d n_{s} / d \ln (k)$ versus $n_{s}$ for various values of $N$. We take $\sim\left(10^{15} \mathrm{GeV}\right), \lambda \sim O\left(10^{68} \mathrm{GeV}^{4}\right), A \sim 10^{-13} M_{p}^{8}$, and $n=2$.

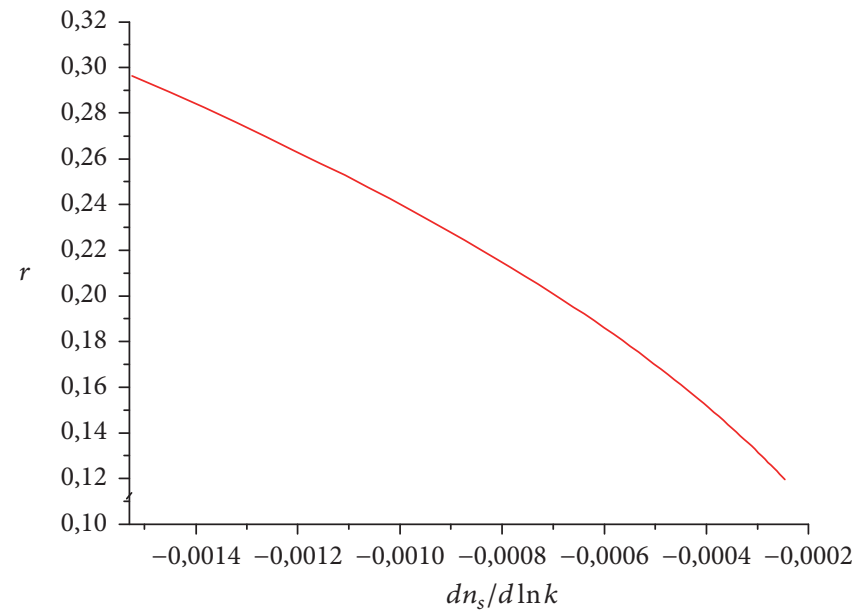

Figure 6: Evolution of $d n_{s} / d \ln (k)$ versus $r$ for various values of $N$. We take $M \sim O\left(10^{15} \mathrm{GeV}\right), \lambda \sim O\left(10^{68} \mathrm{GeV}^{4}\right), A \sim 10^{-13} M_{p}^{8}$, and $n=2$.

data, particularly for a suitable choice of the $e$-folds number $N$. Also, the introduction of parameter $\alpha$ has a small influence on the inflationary parameters.

\section{Conclusion}

In this paper, we have examined the Chaplygin gas model as a candidate for inflation, which showed some important properties. We have considered the original and generalized Chaplygin gas model in the Randall-Sundrum type II braneworld using a monomial potential in the highenergy limit to study the behaviours of inflationary spectrum perturbation parameters. We have found that the inflationary parameters depend on a parameters' space of the model. In the original Chaplygin gas case, $\alpha=1$, we have shown that, for $37<N<60$, the central value of the spectral index is reproduced especially for $n=1 ; 2$ and the running of the spectral index $d n_{s} / d \ln (k)$ is consistent with observations for large interval of $N$. A confrontation with recent Planck data shows that the best fit is achieved for large values of $N$, in particular for the ratio $r$. The case of generalized Chaplygin gas predicts also desirable values of $\left(n_{s} ; r ; d n_{s} / d \ln (k)\right)$ with recent observational data. In particular, the central value, $n_{s} \simeq 0.965$, where $N=65$ corresponds to the value of $\alpha$ in order of $O\left(10^{-3}\right)-O\left(10^{-1}\right)$ and for the ratio $r$ which is consistent with Planck $2015 r<0.11$, is compatible in the case where $N \geq 80$. The values of the tensor-to-scalar ratio $r$ and running of the spectral index $d n_{s} / d \ln (k)$ are in excellent agreement with the latest observations of the Planck satellite for a particular choice of the parameter space of the model.

\section{Conflicts of Interest}

The authors declare that there are no conflicts of interest regarding the publication of this paper.

\section{References}

[1] A. H. Guth, "Inflationary universe: a possible solution to the horizon and flatness problems," Physical Review D: Particles, Fields, Gravitation and Cosmology, vol. 23, no. 2, pp. 347-356, 1981. 
[2] A. D. Linde, Particle Physics and Inflationary Cosmology, vol. 5, Harwood Academic, Chur, Switzerland, 1990.

[3] A. R. Liddle and D. H. Lyth, Cosmological Inflation and LargeScale Structure, Cambridge University Press, Cambridge, UK, 2000.

[4] M. C. Bento, O. Bertolami, and A. A. Sen, "WMAP constraints on the generalized Chaplygin gas model," Physics Letters B, vol. 575, no. 3-4, pp. 172-180, 2003.

[5] A. Aviles, L. Bonanno, O. Luongo, and H. Quevedo, "Holographic dark matter and dark energy with second order invariants," Physical Review D: Particles, Fields, Gravitation and Cosmology, vol. 84, no. 10, Article ID 103520, 2011.

[6] R. Bean and O. Doré, "Are Chaplygin gases serious contenders for the dark energy?" Physical Review D: Particles, Fields, Gravitation and Cosmology, vol. 68, no. 2, Article ID 023515, 2003.

[7] M. Bouhmadi-López and R. Lazkoz, "Chaplygin DGP cosmologies," Physics Letters B: Particle Physics, Nuclear Physics and Cosmology, vol. 654, no. 3-4, pp. 51-57, 2007.

[8] O. Luongo and H. Quevedo, "A unified dark energy model from a Vanishing speed of sound with emergent cosmological Constant," International Journal of Modern Physics D, vol. 23, no. 2, Article ID 1450012, 2014.

[9] L. Xu, Y. Wang, and H. Noh, "Modified Chaplygin gas as a unified dark matter and dark energy model and cosmic constraints," The European Physical Journal C, vol. 72, no. 3, article no. 1931, pp. 1-6, 2012.

[10] M. Jamil, "Interacting new generalized Chaplygin gas," International Journal of Theoretical Physics, vol. 49, no. 1, pp. 62-71, 2010.

[11] J. Gleyzes, D. Langlois, and F. Vernizzi, "A unifying description of dark energy," International Journal of Modern Physics D: Gravitation, Astrophysics, Cosmology, vol. 23, no. 13, Article ID 1443010, 38 pages, 2014.

[12] E. Guendelman, E. Nissimov, and S. Pacheva, "Unified dark energy and dust dark matter dual to quadratic purely kinetic Kessence," The European Physical Journal C, vol. 76, no. 2, 2016.

[13] O. Luongo and H. Quevedo, "An expanding Universe with constant pressure and no cosmological constant," Astrophysics and Space Science, vol. 338, no. 2, pp. 345-349, 2012.

[14] M. Bronstein, Phys. Zeit. Sowejt Union, vol. 3, no. 73, 1993.

[15] M. R. Setare, J. Sadeghi, and A. R. Amani, "Interacting tachyon dark energy in non-flat universe," Physics Letters B, vol. 673, no. 4-5, pp. 241-246, 2009.

[16] P. F. González-Díaz, "Warp drive space-time," Physical Review D: Particles, Fields, Gravitation and Cosmology, vol. 62, no. 4, Article ID 044005, 2000.

[17] E. O. Kahya and B. Pourhassan, "The universe dominated by the extended Chaplygin gas," Modern Physics Letters A, vol. 30, no. 13, Article ID 1550070, 2015.

[18] P. P. Avelino and V. M. C. Ferreira, "Constraints on the dark matter sound speed from galactic scales: the cases of the modified and extended Chaplygin gas," Physical Review D, vol. 91, no. 8, Article ID 083508, 2015.

[19] A. Aviles, A. Bastarrachea-Almodovar, L. Campuzano, and H. Quevedo, "Extending the generalized Chaplygin gas model by using geometrothermodynamics," Physical Review D: Particles, Fields, Gravitation and Cosmology, vol. 86, no. 6, Article ID 063508, 2012.

[20] B. Pourhassan and E. O. Kahya, "FRW cosmology with the extended Chaplygin gas," Advances in High Energy Physics, vol. 2014, Article ID 231452, 11 pages, 2014.
[21] M. Khurshudyan and R. Myrzakulov, "Phase space analysis of some interacting Chaplygin gas models," The European Physical Journal C, vol. 77, no. 2, 2017.

[22] H. B. Benaoum, "Modified Chaplygin gas cosmology with bulk viscosity," International Journal of Modern Physics D, vol. 23, no. 10, Article ID 1450082, 2014.

[23] R. Zarrouki and M. Bennai, "Chaplygin gas braneworld inflation according to WMAP7 data," Physical Review D: Particles, Fields, Gravitation and Cosmology, vol. 82, no. 12, Article ID 123506, 2010.

[24] J. R. Villanueva, “The generalized Chaplygin-Jacobi gas,” Journal of Cosmology and Astroparticle Physics, vol. 2015, no. 7, article no. $045,2015$.

[25] M. C. Bento, O. Bertolami, and A. A. Sen, "Generalized Chaplygin gas, accelerated expansion, and dark-energy-matter unification," Physical Review D: Particles, Fields, Gravitation and Cosmology, vol. 66, Article ID 043507, 2002.

[26] S. B. Nassur, M. J. S. Houndjo, I. G. Salako, and J. Tossa, "Interactions of some fluids with dark energy in $f(T)$ theory," https://arxiv.org/abs/1601.04538.

[27] S. Dutta, S. Mukerji, and S. Chakraborty, "An attempt for an emergent scenario with modified Chaplygin gas," Advances in High Energy Physics, vol. 2016, Article ID 7404218, 5 pages, 2016.

[28] O. Bertolami and V. Duvvuri, "Chaplygin inspired inflation," Physics Letters B, vol. 640, no. 4, pp. 121-125, 2006.

[29] R. Herrera, "Chaplygin inflation on the brane," Physics Letters $B$, vol. 664, no. 3, pp. 149-153, 2008.

[30] R. Herrera, "Tachyon-Chaplygin inflation on the brane," General Relativity and Gravitation, vol. 41, no. 6, pp. 1259-1271, 2009.

[31] Planck Collaboration, "Planck 2015 results. XIII. Cosmological parameters," Astronomy \& Astrophysics, vol. 594, article A13, 63 pages, 2016.

[32] D. H. Lyth and A. Riotto, "Particle physics models of inflation and the cosmological density perturbation," Physics Reports, vol. 314, no. 1-2, pp. 1-146, 1999.

[33] R. Maartens, "Brane-world gravity," Living Reviews in Relativity, vol. 7, article 7, 2004.

[34] D. Langlois, R. Maartens, and D. Wands, "Gravitational waves from inflation on the brane," Physics Letters B, vol. 489, no. 3-4, pp. 259-267, 2000.

[35] P. Brax, C. van de Bruck, and A.-C. Davis, "Brane world cosmology," Reports on Progress in Physics, vol. 67, no. 12, pp. 2183-2231, 2004.

[36] P. Xi and P. Li, "Reexamining generalized Chaplygin gas with the sign-changeable interaction," Astrophysics and Space Science, vol. 360, no. 1, article no. 3, 2015.

[37] L. Randall and R. Sundrum, "Large mass hierarchy from a small extra dimension," Physical Review Letters, vol. 83, no. 17, pp. 3370-3373, 1999.

[38] L. Randall and R. Sundrum, "An alternative to compactification," Physical Review Letters, vol. 83, no. 23, pp. 4690-4693, 1999.

[39] P. Binétruy, C. Deffayet, U. Ellwanger, and D. Langlois, "Brane cosmological evolution in a bulk with cosmological constant," Physics Letters B, vol. 477, no. 1-3, pp. 285-291, 2000.

[40] J. C. Fabris, S. V. B. Gonçalves, and P. E. de Souza, "Letter: density perturbations in a universe dominated by the Chaplygin gas," General Relativity and Gravitation, vol. 34, no. 1, pp. 53-63, 2002.

[41] H. Zhang and Z.-H. Zhu, "Interacting Chaplygin gas," Physical Review D, vol. 73, Article ID 043518, 2006. 
[42] M. J. Reyes-Ibarra and L. A. Ureña-López, "Attractor dynamics of inflationary monomial potentials," AIP Conference Proceedings, vol. 1256, no. 1, p. 293, 2010.

[43] R. Zarrouki, Z. Sakhi, and M. Bennai, "On Braneworld inflation models in light of WMAP7 data," General Relativity and Gravitation, vol. 43, no. 5, pp. 1515-1528, 2011.

[44] B. R. Dinda, S. Kumar, and A. A. Sen, "Inflationary generalized Chaplygin gas and dark energy in light of the Planck and BICEP2 experiments," Physical Review D: Particles, Fields, Gravitation and Cosmology, vol. 90, no. 8, Article ID 083515, 2014.

[45] V. Fayaz, H. Hossienkhani, and A. Jafari, "Effect of anisotropy on the generalized Chaplygin gas scalar field and its interaction with other dark energy models," The European Physical Journal Plus, vol. 132, no. 4, p. 193, 2017.

[46] R. Herrera, M. Olivares, and N. Videla, "Intermediate-generalized Chaplygin gas inflationary universe model," The European Physical Journal C, vol. 73, no. 1, p. 2295, 2013.

[47] A. Jawad, S. Rani, and S. Mohsaneen, "Generalized cosmic Chaplygin inflationary model on the brane," The European Physical Journal Plus, vol. 131, no. 7, article 234, 7 pages, 2016. 

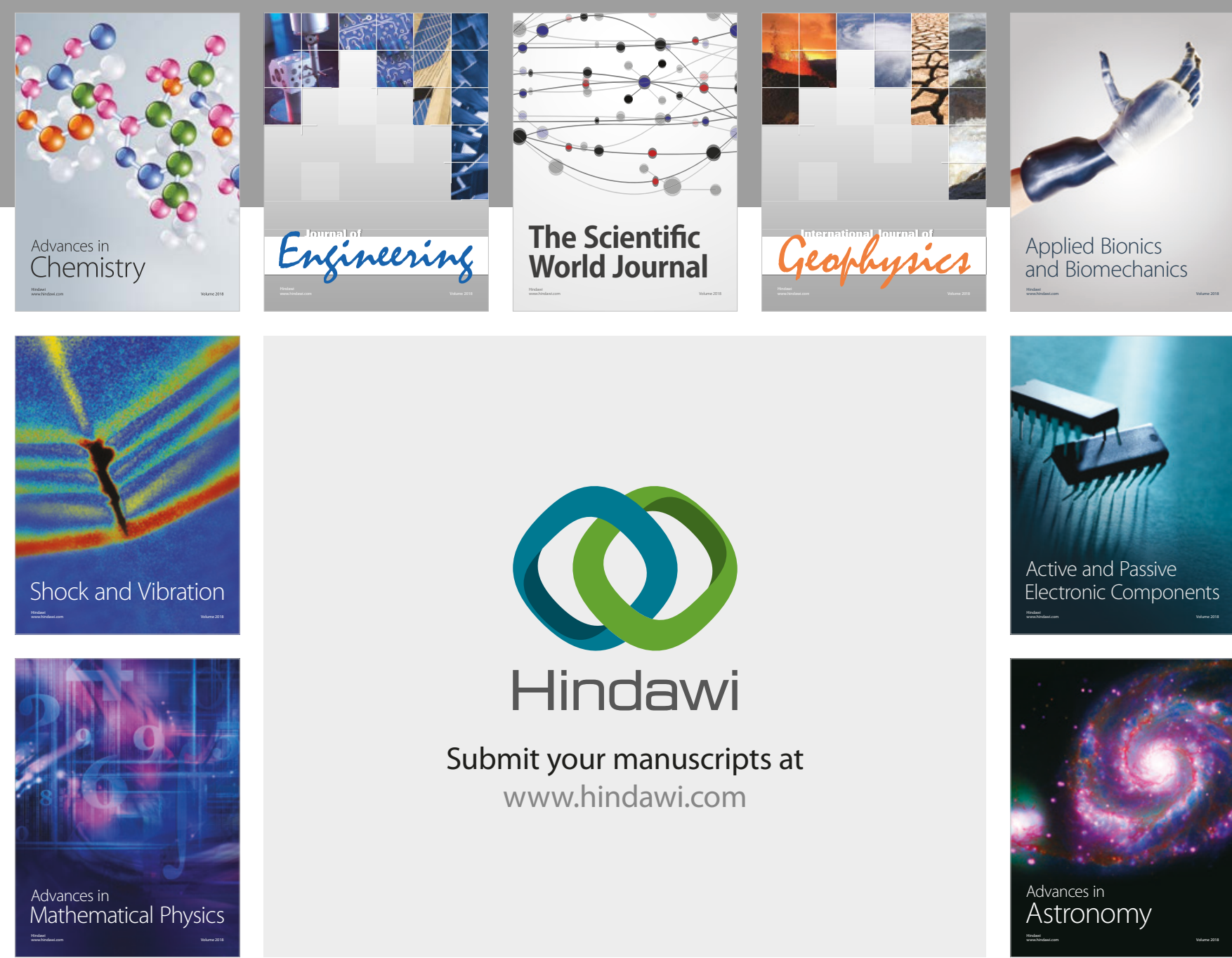

Submit your manuscripts at

www.hindawi.com

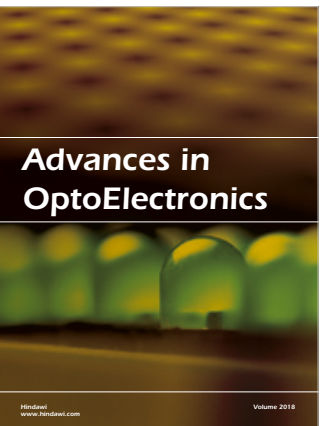

\section{Rotcting Machinery}
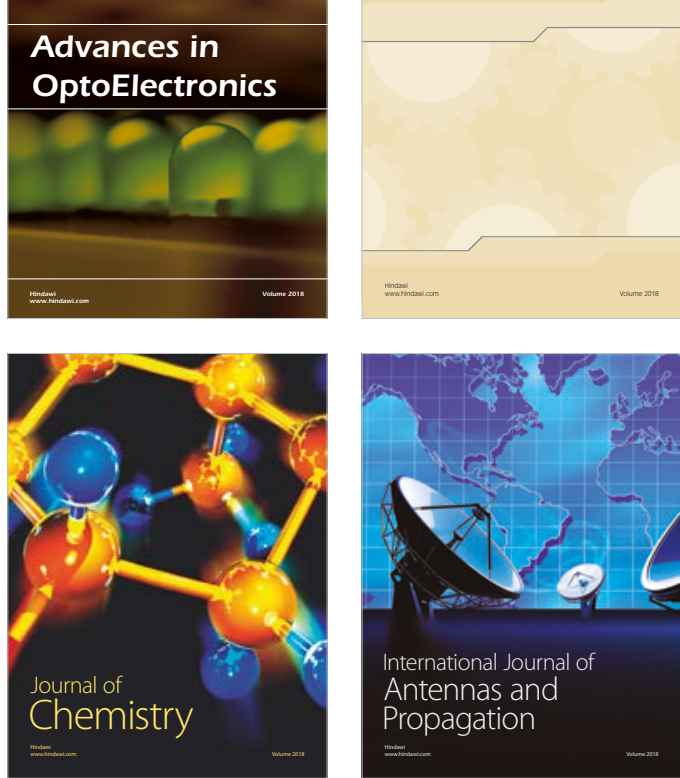

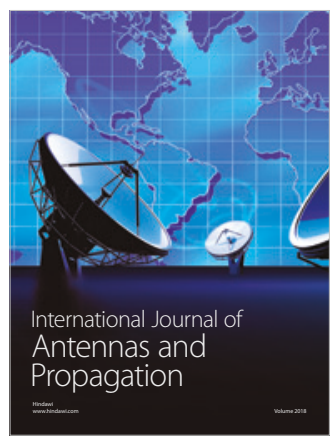

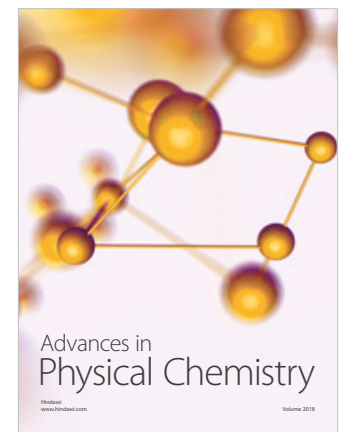

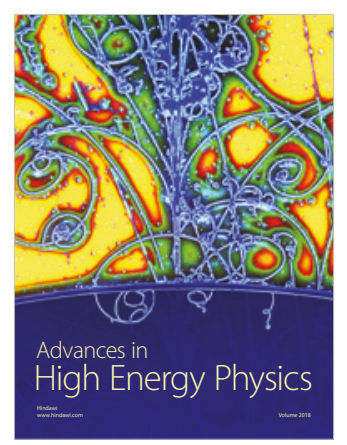

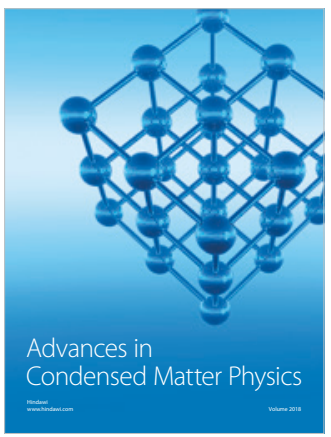

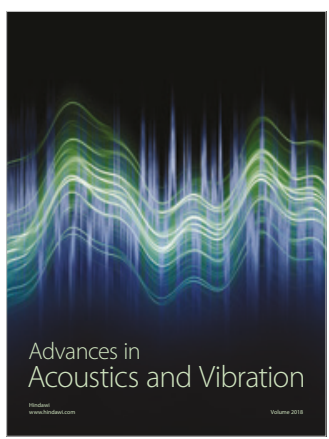

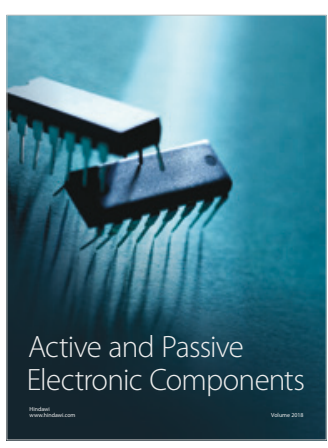
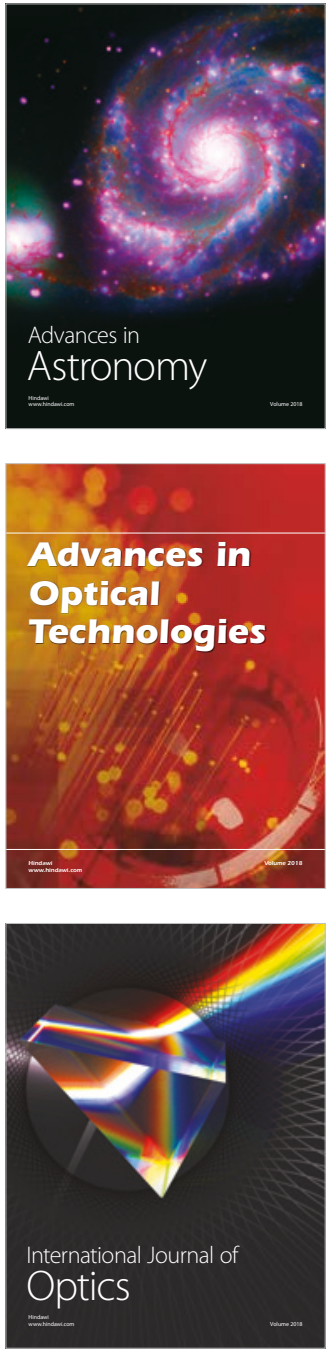